\title{
Aqueous Dispersion and Temperature Induced Reversible Fluorescence Properties of 1-Pyrenecarboxaldehyde
}

\author{
Md. Ashaduzzaman*1, Masashi Kunitake ${ }^{2}$ \\ ${ }^{1}$ Department of Applied Chemistry and Chemical Engineering \\ University of Dhaka, Dhaka 1000, Bangladesh \\ ${ }^{2}$ Department of Nanoscience and Technology, Faculty of New Frontier Sciences, \\ Kumamoto University, 2-39-1 Kurokami, Kumamoto 860-8555, Japan \\ *E-mail address: azaman.du@gmail.com
}

\begin{abstract}
The fluorescence intensities for various vibronic fine structures in the 1-pyrenecarboxaldehyde (PyCHO) fluorescence show strong environment dependence. In aqueous solvent, the distribution of dye is highly depended on its concentration labels and varies from excimer to monomeric state. UVVis Spectroscopic analysis could not able to detect dye below $10^{-7} \mathrm{M}$ concentration, whereas a new monomeric peak at $342 \mathrm{~nm}$ was observed after heating and cooling treatment at above the concentration. At $10^{-8} \mathrm{M}$ concentration, only monomeric distribution of $1-\mathrm{PyCHO}$ reveals a strong temperature $\left(20-50{ }^{\circ} \mathrm{C}\right)$ induced reversible perturbation of the vibronic band intensities. This suggests the operation of some specific solute-solvent dipole-dipole interaction mechanism strongly influenced by heating.
\end{abstract}

Keywords: Monomeric distribution; 1-pyrenecarboxaldehyde; reversible fluorescence

\section{INTRODUCTION}

Investigation of fluorescence probe is becoming an important area in biophysical studies of multimolecular aggregates such as micelles and membranes. Studies with 1-PyCHO as a fluorescence probe have received special consideration, because 1-PyCHO has several interesting photophysical properties which make it suitable for use as an effective probe, notably the long life-time of 1-PyCHO monomers and efficient formation of excimers. 1$\mathrm{PyCHO}$ is one of the derivatives of pyrene structure.

There have been extensive studies on the photophysics of pyrene: its electronic spectrum and state assignments ${ }^{1}$, kinetic details of excimer formation ${ }^{2}$, spectral pressure effects $^{3}$, formation and kinetics of excited state $^{4}$, photoionization ${ }^{5}$, delayed luminescence ${ }^{6}$, and quasilinear spectra ${ }^{7}$, yet there have been few studies directed toward environmental effects on the fluorescence spectrum of pyrene.

The solvent dependence of vibronic band intensities in pyrene monomer fluorescence was first investigated by Nakajima ${ }^{8-11}$. The intensities of the various vibronic bands were found to show a strong dependence on the solvent environment.

Recently, pyrene has been using as a labeled probe with polymers to investigate their molecular properties $1^{12-14}$. Winnik and his colleagues ${ }^{14}$ have reported that aqueous solutions of the pyrene-labeled poly(ethylenimine) (PEI-Py) exhibit typical pyrene monomer and 
excimer emission, and the excimer - to - monomer ratio $\left(I_{\mathrm{E}} / I_{\mathrm{M}}\right)$ decreases with decreasing Py content of the macromolecule. The excimer emission arises from preassociated pyrene aggregates. Introduction of $-\mathrm{CHO}$ with pyrene structure makes it a little polar character, which facilitate to dissolve in aqueous solution.

Mazur and Blanchard ${ }^{15}$ have reported that the density of pyrene onto the quartz surface produced monomeric and excimer spectra.

Generally, depending on concentration the free probes reveal monomeric or excimer fluorescence spectra in solution. Excimer formation and their fluorescence intensities are not consistent at all. Therefore, the study of 1-PyCHO probe regarding monomeric species is very important to explore unique characteristic in aqueous solution. In this article, we report the temperature induced perturbation of vibronic bands intensities of monomeric 1-PyCHO in aqueous solution.

\section{EXPERIMENTAL}

\section{1. Materials and method}

Fluorescence measurements of 1pyrenecarboxaldehyde were recorded by using a FP6500 Spectrofluorometer (serial no. 0041100523, model: FP-6500DS-A) equipped with a heater (Advantec, model: LCH-3000, serial no. 201061, Tokyo Kaisha Ltd, Japan). Microliters of 1-PyCHO stock acetone solutions were first evaporated in vials and diluted with milli-Q water, yielding ca. 1.0-0.01 $\mu \mathrm{M}$ concentrations of aqueous solution.

The thermo-responsive behavior of 1-PyCHO was investigated by means of a UV-Vis

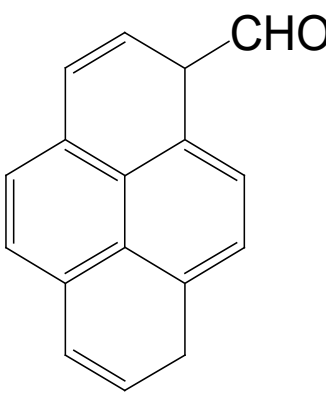

1-Pyrenecarboxaldehyde Spectrophotometer (JASCO V-650 Spectrophotometer, serial no. A004061150, Japan).

\section{RESULTS AND DISCUSSION}

At the very beginning, we started to investigate the monomeric level of concentration of 1-PyCHO in aqueous solution and the influence of sonication and temperature on UV-Vis spectrophotometric absorption and fluorometric excitation and emission spectra of the dye.

In UV-Vis spectrophotometric absorption, it was revealed that $1.0 \mu \mathrm{M}$ aqueous dye solution persisted aggregation along with dimer or multimer peaks at $\lambda_{\max } 365.5 \mathrm{~nm}$, as shown in Fig. 1. The spectrum recorded after 5 minutes sonication showed an additional peak at $\lambda_{\max }$ $342 \mathrm{~nm}$, indicates disintegration of dye in the solution.

When the same solution was heated at $50{ }^{\circ} \mathrm{C}$ and then subsequently cooled to room temperature $\left(25^{\circ} \mathrm{C}\right)$, the peak at $\lambda_{\max } 365.5 \mathrm{~nm}$ was almost diminished although the new peak at $\lambda_{\max } 342 \mathrm{~nm}$ with a shoulder at $\lambda_{\max } 325 \mathrm{~nm}$ became prominent which also verify the reduction of dye into smaller fragment. 


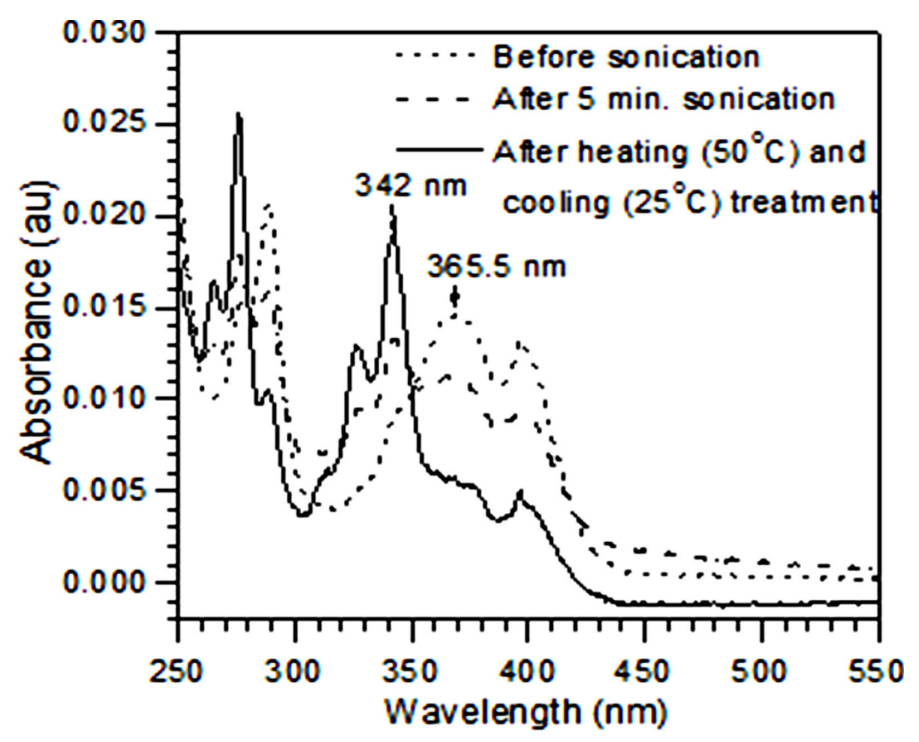

Fig. 1. UV-vis spectra of $1 \times 10^{-6} \mathrm{M}$

$1-\mathrm{PyCHO}$ aqueous solutions

However, when similar experiments were performed using $0.1 \mu \mathrm{M}$ and $0.01 \mu \mathrm{M}$ aqueous solutions shown in Fig. 2 (a, b), an experimental problem was arisen to measure $0.01 \mu \mathrm{M}$ solution because of very low concentration of dye. Besides, $0.1 \mu \mathrm{M}$ solution showed only two peaks at $\lambda_{\max } 342 \mathrm{~nm}$ and at $\lambda_{\max } 325 \mathrm{~nm}$ representing monomeric species of the dye. On the other hand, more precise investigation with fluorometric spectra was conducted. The original molecular states (monomeric and aggregation) of the dye in aqueous solution was observed as it was excited from two different wavelengths at $\lambda_{\max } 342 \mathrm{~nm} \& 365.5 \mathrm{~nm}$ and recorded emission spectra.

When $1 \mu \mathrm{M}$ aqueous solution of 1-PyCHO dye after 5 minutes sonication was excited at $\lambda_{\max } 365.5 \mathrm{~nm}$ an excimer emission spectrum was obtained at $\lambda_{\max } 472 \mathrm{~nm}$ without any evidence of monomeric peak at $\lambda_{\max } 382 \mathrm{~nm}$, besides two emission peaks were found at $\lambda_{\max }$ $382 \mathrm{~nm}$ and $472 \mathrm{~nm}$ as excited the same solution at $\lambda_{\max } 342 \mathrm{~nm}$ indicates along with the excimer some monomeric species were formed is shown in Fig. $3(a, b)$.
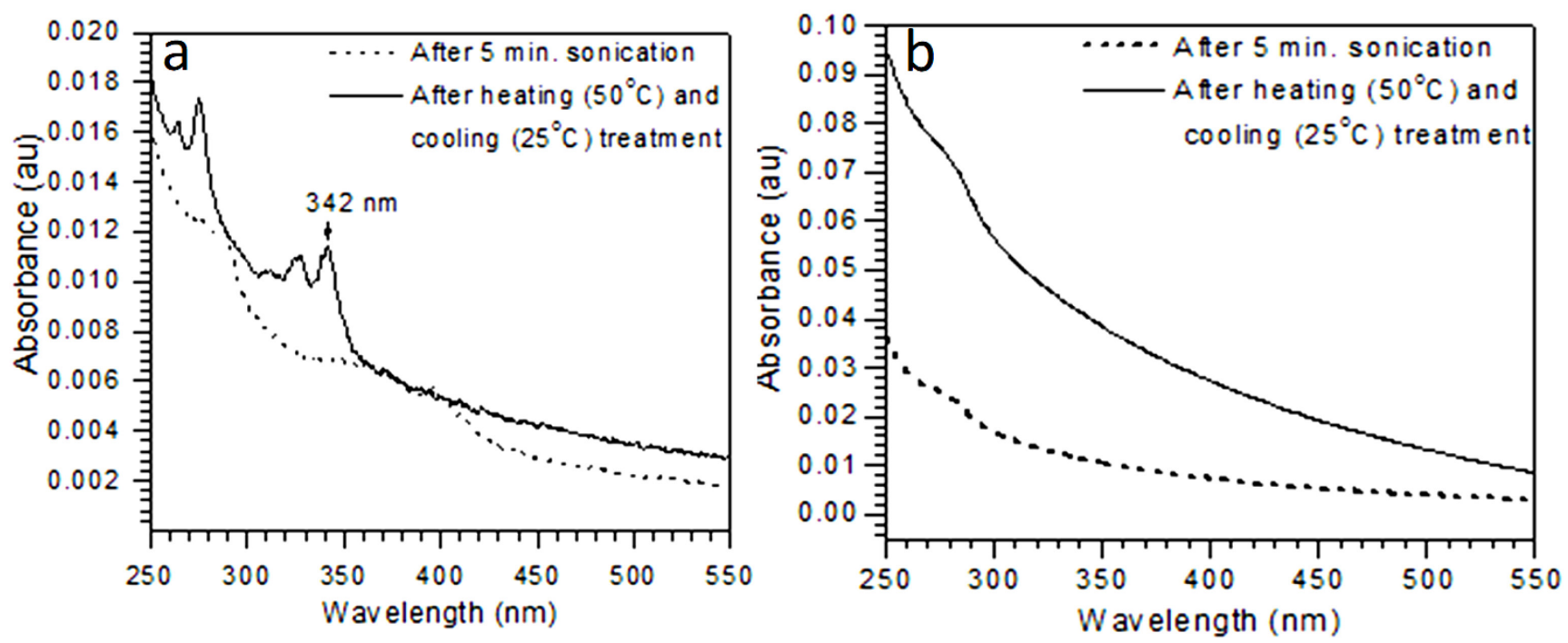

Fig. 2. UV-vis spectra of $1 \times 10^{-6} \mathrm{M}$ (A) and $1 \times 10^{-7} \mathrm{M}$ (B) $1-\mathrm{PyCHO}$ aqueous solutions 
Solution of $0.1 \mu \mathrm{M}$ 1-PyCHO showed excitation and emission spectra with resemblance of $1 \mu \mathrm{M}$ PyCHO solution only giving very low peak intensity at $\lambda_{\max } 382 \mathrm{~nm}$ and a quenched excimer peak at $\lambda_{\max } 472 \mathrm{~nm}$ when excited at $\lambda_{\max } 365.5 \mathrm{~nm}$ and $342 \mathrm{~nm}$ respectively as shown in Fig. 3 (c, d). In Fig. 3 (e, f), the more diluted solution $0.01 \mu \mathrm{M}$ 1-PyCHO was unable to detect peak by UV-Vis spectrophotometer, gave an excellent emission spectra by fluorometry at $\lambda_{\max } 382 \mathrm{~nm}$ with a mirror image of excited spectra at $\lambda_{\max } 342 \mathrm{~nm}$ having a shoulder at $\lambda_{\max } 400 \mathrm{~nm}$ indicates the monomeric form of dye in aqueous solution.
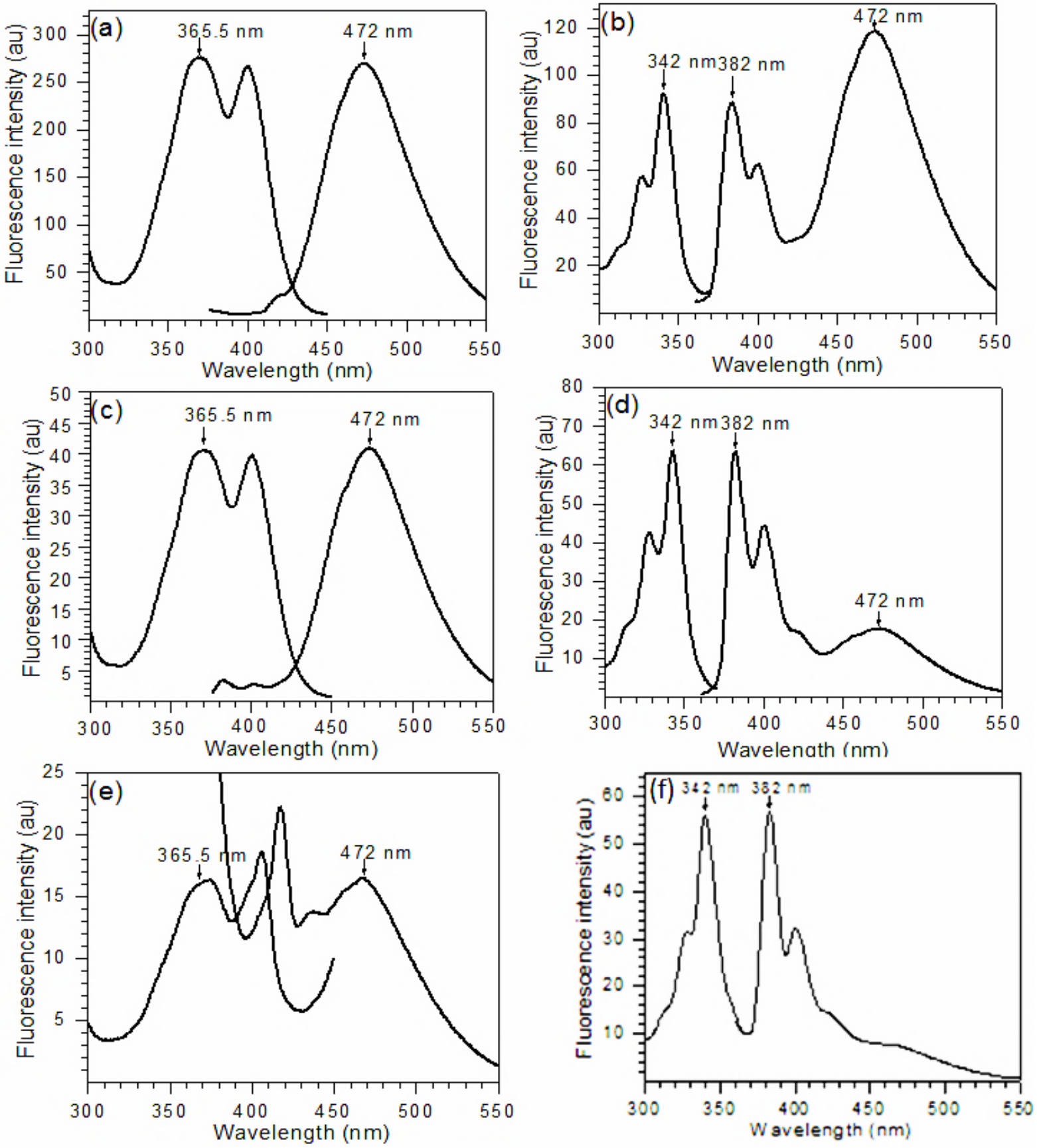

Fig. 3. Spectrofluorometric analysis of 1-pyrenecarboxaldehyde at different concentrations (a, b) $1.0 \times 10^{-6} \mathrm{M},(\mathrm{c}, \mathrm{d}) 1.0 \times 10^{-7} \mathrm{M}$ and (e, f) $1.0 \times 10^{-8} \mathrm{M}$ where excitation and emission spectra were recorded at $365.5 \mathrm{~nm}$ and $472 \mathrm{~nm}$ for excimer (a, c, e) and $342 \mathrm{~nm}$ and $382 \mathrm{~nm}$ for monomer (b,

$\mathrm{d}, \mathrm{f})$ respectively 
Aqueous dispersion of 1-PyCHO depending on concentration is schematically presented in Fig. 4 and the summary of instrumental responses is given in Table 1.

Table 1. Aqueous dispersion of 1-pyrenecarboxaldehyde fluorescence dye at different concentrations with instrumental responses.

\begin{tabular}{ccccc}
\hline Dispersion & $\begin{array}{c}\text { Monomeric } \\
\text { dispersion }\end{array}$ & $\begin{array}{c}\text { Excimer/Monomeric } \\
\text { dispersion }\end{array}$ & $\begin{array}{c}\text { Aggregated } \\
\text { dispersion }\end{array}$ & Precipitation \\
\hline Concentration & $1.0 \times 10^{-8} \mathrm{M}$ & $1.0 \times 10^{-7} \mathrm{M}$ & $1.0 \times 10^{-6} \mathrm{M}$ & $>1.0 \times 10^{-6} \mathrm{M}$ \\
\hline $\begin{array}{c}\text { UV-Vis } \\
\text { spectroscopy }\end{array}$ & $\times$ & 0 & 0 & $\times$ \\
\hline Spectrofluorometry & 0 & 0 & 0 & $\times$ \\
\hline
\end{tabular}

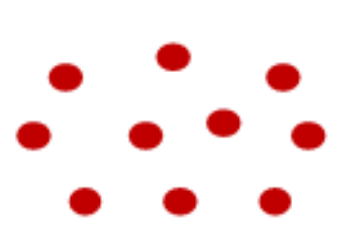

Monomeric dispersion

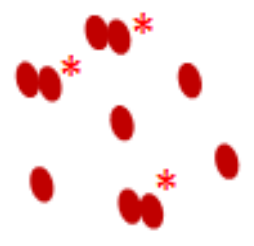

Excited excimer

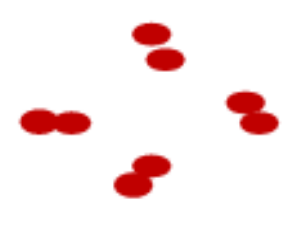

Dispersion aggregate

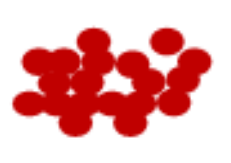

Precipitate

Fig. 4. Schematic presentation of aqueous dispersion of 1-pyrenecarboxaldehyde fluorescence dye.

The solvent-dependent fluorescence behavior of aromatic aldehydes, including PyCHO has been reported by Brederick et $\mathrm{al}^{16}$. In nonpolar solvents such as $n$-heptane, the fluorescence spectrum of $\mathrm{PyCHO}$ is structured with peaks around 400-420 nm, and the quantum yield $\left(\phi_{\mathrm{F}}<0.001\right)$ is small.

This particular fluorescence is due to an $n-\pi^{*}$ transition, however, on increasing the polarity of the solvent media, the $\pi-\pi^{*}$ level by solvent relaxation during the lifetime of the excited state.

Thus in polar solvents the $\pi-\pi^{*}$ state becomes the fluorescence emitting state. Presumably a solvent environment with dielectric constant ca. 10 is required to equalize the $n$ $\pi^{*}$ and $\pi-\pi^{*}$ levels. The monomeric fluorescence of PyCHO shows a strong dependence on solvent polarity has also been reported by Kalyanasundaram et al ${ }^{17}$.

The fluorescence in nonpolar solvents such as $n$-hexane is very weak (quantum yield $<0.001)$, but becomes quite intense in polar solvents $\left(\phi_{\mathrm{F}}\right.$ in methanol $\left.=0.15\right)$.

Water as a polar solvent, $\pi-\pi^{*}$ state would be the fluorescence emitting state where, water molecules essentially surrounded the PyCHO molecules with hydrogen bonds. It is noteworthy to mention that at low temperature, the entropy of water molecules is lower than the entropy in boiling water. 


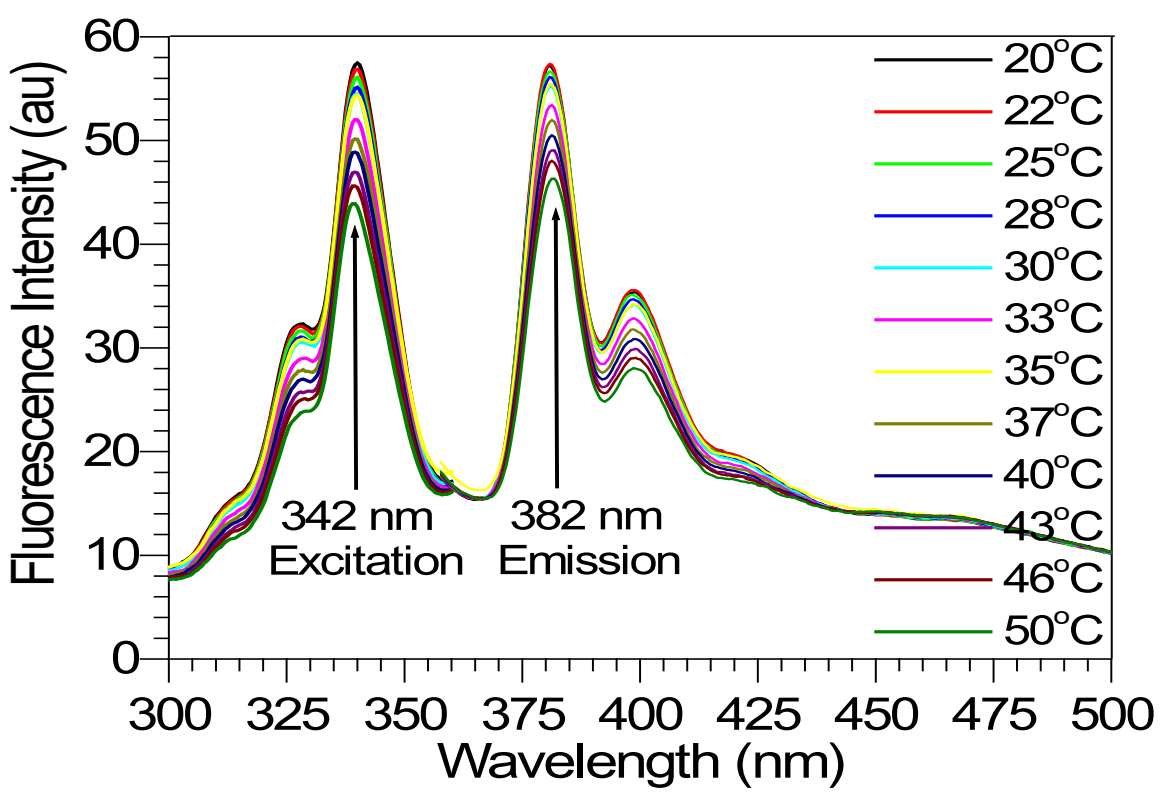

Fig. 5. The spectra for the variation of fluorescence intensities, when temperature was gradually increased from $20^{\circ} \mathrm{C}$ to $50^{\circ} \mathrm{C}$ at a heating rate of $1^{\circ} \mathrm{C} / \mathrm{min}$.

Fig. 5, represents the spectra for the variation of fluorescence intensities, when temperature was gradually increased from $20{ }^{\circ} \mathrm{C}$ to $50{ }^{\circ} \mathrm{C}$ at a heating rate of $1{ }^{\circ} \mathrm{C} / \mathrm{min}$. The solution was excited at $342 \mathrm{~nm}$ wavelength, emission spectrum at $382 \mathrm{~nm}$ was obtained as we

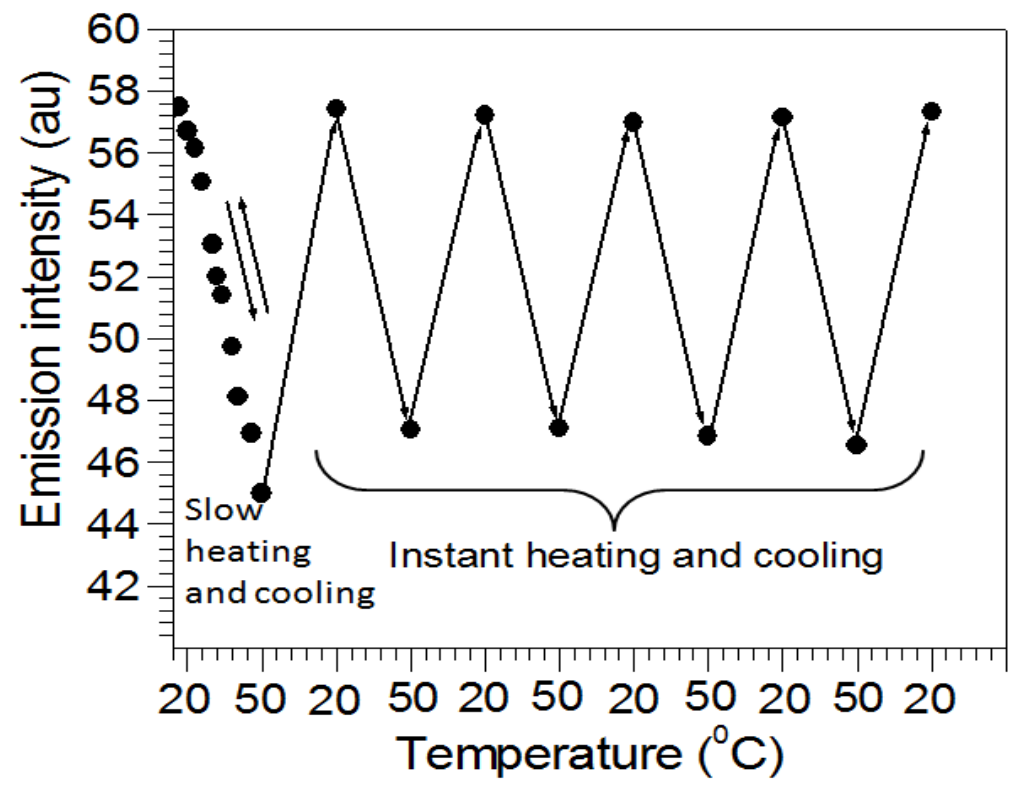

Fig. 6. The influence of temperature on monomeric fluorescence intensities of 1 $\mathrm{PyCHO}$ in water at $10^{-8} \mathrm{M}$ concentration during slow and rapid heating. 
mentioned earlier. The shoulder at $400 \mathrm{~nm}$ of the emission spectra were more pronounced than the shoulder at $325 \mathrm{~nm}$ of excited spectra. During heating and cooling operation, the maxima at $382 \mathrm{~nm}$ of emission spectra were remained unchanged, indicates no excimer was grown in the solution at $10^{-8} \mathrm{M}$ concentration.

Fig. 6 reveals the intensities change for emission spectra. The intensity was varied steadily and reversibly (linear dotted line) between ca.44 au and ca.58 au during slow heating and cooling process.

On the other hand, in rapid heating process, cell solution was dipped into bath water which was preheated at $50{ }^{\circ} \mathrm{C}$ and kept for 3 minutes to raise the desired temperature; spectra were then recorded at $50{ }^{\circ} \mathrm{C}$ temperature from a preset spectrofluometer. At $20{ }^{\circ} \mathrm{C}$, the intensities were recorded ca. 58 au, but at $50{ }^{\circ} \mathrm{C}$ temperature, emission intensities ca 46 are also very close to ca. $44 \mathrm{au}$, as we obtained from slow heating process. It is expected that the fluorescence intensity is sensitive to the change of temperature because it affects the viscosity of the medium and hence the number of collisions of the molecules of dye with solvent molecules and the strength of hydrogen bonding.

\section{CONCLUSION}

Investigation of aqueous monomeric distribution of 1-pyrenecarboxaldehyde and its reversible tuning properties with temperature would be crucial for direct surface modification of substrate to use in the fields of medical, electro-chemical as well as industrial applications.

\section{ACKNOWLEDGEMENT}

We are gratefully acknowledge support from the Graduate School Action Scheme for the Internationalization of University Students (GRASIUS) program of the Japanese Ministry of Education, Culture, Sports, Science and Technology.

\section{References}

[1] P. A. Geldof., R. P. H. Rettschnick, G. J. Hoytink, Chemistry of Physics Letter 4 (1969) 59-61.

[2] J. B. Binks, Wiley-Interscience, New York, N.Y., 1970.

[3] H. W. Offen in, Vol. I, J. B. Birks, Ed., Wiley-Interscience. New York, N.Y., 1975.

[4] J. T. Richards, A. West, J. K. Thomas, Journal of Physical Chemistry 74 (1970) 4137.

[5] M. Gratzel, J. K. Thomas, Journal of Physical Chemistry 78 (1974) 2208.

[6] C. A. Parker, Elsevier. New York, N.Y., 1968.

[7] A. Pellois, J. Rlpoche, Chemistry of Physics Letter 3 (1989) 280.

[8] A. Nakajima, Bulletin of Chemical Society of Japan 44 (1971) 3272.

[9] A. Nakajima, Spectrochim. Acta, Part A, 30 (1974) 860.

[10] A. Nakajima, Journal of Molecular Spectroscopy 61 (1976) 467.

[11] A. Nakajima, Jornal of Luminescence 11 (1976) 429. 
[12] J. Rao, J. Xu, S. Luo, S. Liu, Langmuir 23 (2007) 11857-11865.

[13] R. D. Stramel, Chinh Nguyen, S . E. Webber, M. A. J. Rodgers, J. Phys. Chem., 92 (1988) 2934-2938.

[14] M. A. Winnik, S. M. Bystryak, Z. Liu, J. Siddiqui, Macromolecules 31 (1998) 6864.

[15] M. Mazur, G. J. Blanchard, Journal of Phyical Chemistry B 109 ( 2005) 4076-4083.

[16] K. Bredereck, Th. Forster, H.G. Oenstein, Ed. Willey, New York, N.Y., 1960.

[17] K. Kalyanasundaram, J. K. Thomas, Journal of Physical Chemistry 81 (1977) 23. 